Copyright ( 2011 IEEE. Personal use of this material is permitted. Permission from IEEE must be obtained for all other uses, in any current or future media, including reprinting/republishing this material for advertising or promotional purposes, creating new collective works, for resale or redistribution to servers or lists, or reuse of any copyrighted component of this work in other works. 


\title{
Lead-Lag compensators: analytical and graphical design on the Nyquist plane
}

\author{
Roberto Zanasi, Stefania Cuoghi and Lorenzo Ntogramatzidis
}

\begin{abstract}
This paper presents a design method for the parameters of a general class of lead-lag compensators to exactly satisfy gain and phase margins specifications. A free parameter of the compensator is used as a degree of freedom in order to satisfy additional requirements on the phase and/or gain crossover frequencies. A graphical procedure for the compensator design on the Nyquist plane is presented with some numerical examples.
\end{abstract}

\section{INTRODUCTION}

From classical control theory, it is known that the gain and phase margins (GPM) serve as important measures of the robustness of a dynamical system. The phase margin is related to the damping of the system, and therefore also serves as performance measurement [1]. Different methods can be found in the literature to satisfy GPM specifications [2][7]. However such solutions are usually obtained with trial-and-error methods. In 1998 a graphical method for the design of lead-lag compensators to satisfy GPM specifications was presented [2]. The recent literature shows a renewed interest in the design of classical controllers [8]-[10]. In this paper we propose both a numerical and a graphical solution to the design of lead-lag compensators on GPM specifications. We utilize a general structure for the lead-lag compensator with real and complex zeros and poles, but we relate it to the classical one with real zeros and poles. The paper is organized as follows: in Section II, the basic properties of this general form of lead-lag compensator are presented. In Section III the inversion formulae and their properties are described. In Section IV the design problems with constraints on the GPM are introduced, and their solutions is presented along with a graphical representation. Numerical examples and conclusions end the paper.

This work was partially supported by the Australian Research Council (Discovery Grant DP0986577).

R. Zanasi and S. Cuoghi are with the Faculty of Engineering, DII-Information Engineering Department, University of Modena and Reggio Emilia Via Vignolese 905, 41100, Modena, Italy, e-mails: roberto.zanasi@unimore.it and stefania.cuoghi@unimore.it.

L. Ntogramatzidis is with the Department of Mathematics and Statistics, Curtin University, Perth, WA, Australia, e-mail: L. Ntogramatzidisecurtin.edu.au.

\section{LEAD-LAG COMPENSATORS: THE GENERAL STRUCTURE}

Consider a lead-lag compensator described by the transfer function

$$
C(s)=\frac{s^{2}+2 \gamma \delta \omega_{n} s+\omega_{n}^{2}}{s^{2}+2 \delta \omega_{n} s+\omega_{n}^{2}},
$$

where $\gamma, \delta$ and $\omega_{n}$ are real and positive. When $\gamma \delta<1$ and/or $\delta<1$ the zeros and/or the poles of the lead-lag compensator $C(s)$ are complex conjugate with negative real part. The compensator $C(s)$ has a unity static gain $C(0)=1$ which does not change the static behavior of the controlled system. Notice that the compensator $\mathrm{C}(\mathrm{s})$ is written in a general form which encompasses the classical lead-lag form $C_{r}(s)$ with real poles and real zeros:

$$
C_{r}(s)=\frac{\left(1+\tau_{1} s\right)\left(1+\tau_{2} s\right)}{\left(1+\alpha \tau_{1} s\right)\left(1+\frac{\tau_{2}}{\alpha} s\right)}
$$

where $0<\tau_{1}<\tau_{2}$ and $0<\alpha<1$. The relations that link parameters in (1) and (2) are:

$$
\begin{gathered}
\tau_{1}=\frac{\gamma \delta-\sqrt{\gamma^{2} \delta^{2}-1}}{\omega_{n}}, \quad \tau_{2}=\frac{\gamma \delta+\sqrt{\gamma^{2} \delta^{2}-1}}{\omega_{n}}, \\
\alpha=\frac{\delta-\sqrt{\delta^{2}-1}}{\gamma \delta-\sqrt{\gamma^{2} \delta^{2}-1}} .
\end{gathered}
$$

The frequency response of compensator $C(s)$ is

$$
C(j \omega)=\frac{\omega_{n}^{2}-\omega^{2}+j 2 \gamma \delta \omega_{n} \omega}{\omega_{n}^{2}-\omega^{2}+j 2 \delta \omega_{n} \omega},
$$

which can also be written for $\omega \neq \omega_{n}$ as:

$$
C(j \omega)=\left.\frac{1+j X(\omega)}{1+j Y(\omega)}\right|_{\omega \neq \omega_{n}},
$$

where

$$
X(\omega)=\frac{2 \gamma \delta \omega \omega_{n}}{\omega_{n}^{2}-\omega^{2}}, \quad Y(\omega)=\frac{2 \delta \omega \omega_{n}}{\omega_{n}^{2}-\omega^{2}} .
$$

Since $\gamma, \delta$ and $\omega_{n}$ are assumed to be real and positive, $X(\omega)$ and $Y(\omega)$ satisfy

$$
\left\{\begin{array}{lll}
X(\omega)>0, Y(\omega)>0 & \text { when } & \omega<\omega_{n}, \\
X(\omega)<0, Y(\omega)<0 & \text { when } & \omega>\omega_{n} .
\end{array}\right.
$$

The parameter $\gamma$ is the gain of $C(j \omega)$ at frequency $\omega=\omega_{n}$. From (3) and (4) we get:

$$
\gamma=C\left(j \omega_{n}\right)=\left.\frac{X(\omega)}{Y(\omega)}\right|_{\omega \neq \omega_{n}} .
$$




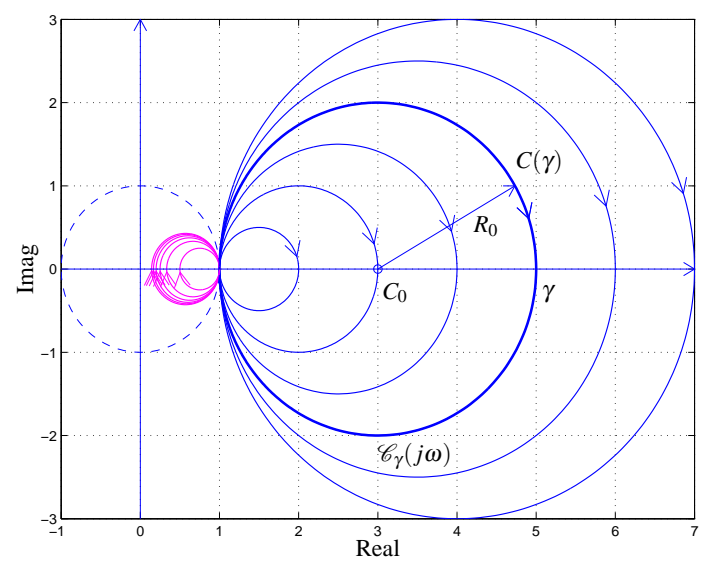

Fig. 1. Nyquist diagrams of $C(j \omega)$ when $\omega_{n}=1,(\delta=1.5$, $\gamma=[2: 1: 7]$, blue lines) and $(\gamma \delta=1.5, \gamma=1 . /[2: 1: 7]$, magenta lines). The thick blue line corresponds to $\delta=1.5$ and $\gamma=5$.

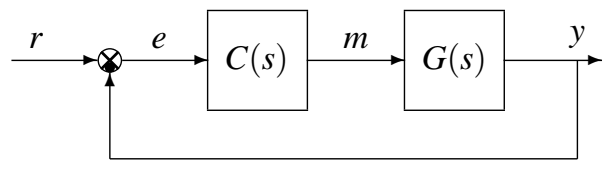

Fig. 2. Unity feedback control structure

The gain $\gamma$ is the minimum (or maximum) amplitude of $C(j \omega)$. The Nyquist diagram of $C(j \omega)$ for $\omega_{n}=1$ and for different values of parameters $\delta$ and $\gamma$ is shown in Fig. 1. The shape of these diagrams are circles, as the following property explains.

Definition 1: let $\mathscr{C}(\gamma)$ denote the set of all the lead-lag compensators $C(s)$ as defined in (1) having the same parameter $\gamma$, that is

$$
\mathscr{C}(\gamma)=\left\{C(s) \text { as in (1) } \mid \delta>0, \omega_{n}>0\right\} .
$$

Moreover, let $\mathscr{C}_{\gamma}(s) \in \mathscr{C}(\gamma)$ denote one element of set $\mathscr{C}(\gamma)$ chosen arbitrarily.

Property 1: the shape of the frequency response $\mathscr{C}_{\gamma}(j \omega)$ of $\mathscr{C}_{\gamma}(s)$ on the Nyquist plane, see Fig. 1 , is a circle with center $C_{0}$ and radius $R_{0}$

$$
C(\gamma)=C_{0}+R_{0} e^{j \theta}, \quad C_{0}=\frac{\gamma+1}{2}, \quad R_{0}=\frac{|\gamma-1|}{2},
$$

where $\theta \in[0,2 \pi]$. The intersections of $\mathscr{C}_{\gamma}(j \omega)$ with the real axis occur at points 1 and $\gamma$. The shape does not depend on $\delta>0$ and $\omega_{n}>0$.

Proof: It can be easily proved that the distance $d=\left|\mathscr{C}_{\gamma}(j \omega)-C_{0}\right|$ of the generic point $\mathscr{C}_{\gamma}(j \omega)$ from the center $C_{0}$ is constant and equal to radius $R_{0}$. From (3) it is seen that variations of $\omega_{n}$ and $\delta$ modify the distribution of frequency $\omega$ on the Nyquist diagram of $\mathscr{C}_{\gamma}(j \omega)$, but they do not change the diagram shape, which only depends on $\gamma$.

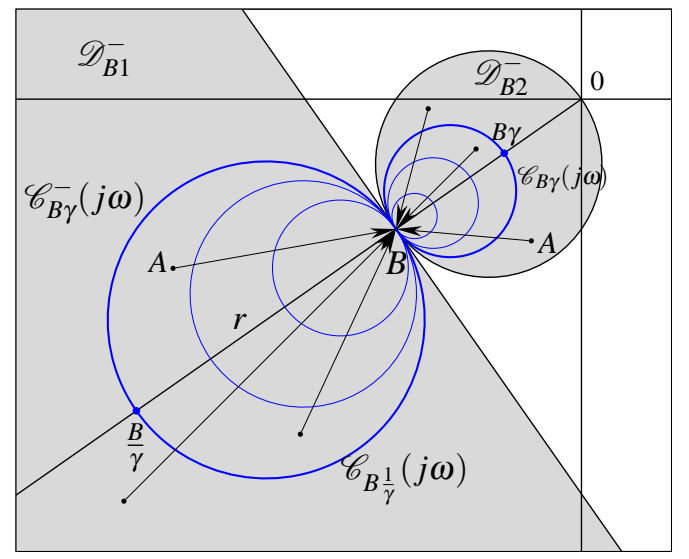

Fig. 3. Controllable domain $\mathscr{D}_{B}^{-}=\mathscr{D}_{B 1}^{-} \cup \mathscr{D}_{B 2}^{-}$of lead-lag compensator $C(s)$ on the Nyquist plane.

\section{LEAD-LAG COMPENSATORS $C\left(s, \omega_{n}\right)$ MOVING A POINT $A$ TO A POINT $B$}

Consider the block-diagram shown in Fig. 2, where $G(s)$ denotes the transfer function of the LTI plant to be controlled, which may include the gain and the integration terms required to meet the steady-state accuracy specifications. The lead-lag compensator $C(s)$ has to be designed in order to satisfy phase margin $\phi_{m}$ and gain margin $G_{m}$ specifications. Let $C\left(j \omega_{0}\right)=M_{0} e^{j \varphi_{0}}$ denote the value of the frequency response of the compensator $C(s)$ at frequency $\omega_{0}$, where $M_{0}=M\left(\omega_{0}\right)$ and $\varphi_{0}=\varphi\left(\omega_{0}\right)$. To study how $C\left(j \omega_{0}\right)$ affects $G(j \omega)$ at frequency $\omega_{0}$, let us consider two generic points $A=M_{A} e^{j \varphi_{A}}$ and $B=M_{B} e^{j \varphi_{B}}$ of the complex plane. Referring to Fig. 3, we say that point $A$ is controllable to point $B$ (or equivalently that point $A$ can be moved to point $B)$ if a value $C\left(j \omega_{0}\right)$ exists such that

$$
B=C\left(j \omega_{0}\right) \cdot A,
$$

that is if and only if the following conditions hold:

$$
M_{B}=M_{A} M_{0}, \quad \varphi_{B}=\varphi_{A}+\varphi_{0} .
$$

Definition 2: $\left(\mathscr{D}_{B}^{-}\right)$given a point $B \in \mathbb{C}$, let us define "controllable domain of lead-lag compensator $C(s)$ to point $B$ " as

$\mathscr{D}_{B}^{-}=\left\{A \in \mathbb{C} \mid \exists \gamma, \delta, \omega_{n}>0, \exists \omega \geq 0: C(j \omega) \cdot A=B\right\}$

It is easy to see that domain $\mathscr{D}_{B}^{-}$can be expressed, see Fig. 3, as $\mathscr{D}_{B}^{-}=\mathscr{D}_{B 1}^{-} \cup \mathscr{D}_{B 2}^{-}$where

$$
\begin{aligned}
\mathscr{D}_{B 1}^{-}= & \left\{A=M_{A} e^{j \varphi_{A}} \mid-\frac{\pi}{2}+\varphi_{B}<\varphi_{A}<\frac{\pi}{2}+\varphi_{B},\right. \\
& \left.M_{A}>\frac{M_{B}}{\cos \left(\varphi_{A}-\varphi_{B}\right)}\right\}, \\
\mathscr{D}_{B 2}^{-}= & \left\{A=M_{A} e^{j \varphi_{A}} \mid-\frac{\pi}{2}+\varphi_{B}<\varphi_{A}<\frac{\pi}{2}+\varphi_{B},\right. \\
& \left.0<M_{A}<M_{B} \cos \left(\varphi_{A}-\varphi_{B}\right)\right\} .
\end{aligned}
$$


Definition 3: given a point $B \in \mathbb{C}$, let $\mathscr{C}_{B}(\gamma)$ and $\mathscr{C}_{B}^{-}(\gamma)$ denote the sets of lead-lag compensators $C(s)$ defined as follows

$$
\begin{aligned}
& \mathscr{C}_{B}(\gamma)=\{B \cdot C(s) \mid C(s) \in \mathscr{C}(\gamma)\} \\
& \mathscr{C}_{B}^{-}(\gamma)=\left\{\frac{B}{C(s)} \mid C(s) \in \mathscr{C}(\gamma)\right\}
\end{aligned}
$$

with $\mathscr{C}(\gamma)$ defined in (8). Moreover, let $\mathscr{C}_{B \gamma}(s) \in$ $\mathscr{C}_{B}(\gamma)$ and $\mathscr{C}_{B \gamma}^{-}(s) \in \mathscr{C}_{B}^{-}(\gamma)$ denote particular elements of the two sets $\mathscr{C}_{B}(\gamma)$ and $\mathscr{C}_{B}^{-}(\gamma)$ chosen arbitrarily.

Property 2: given $\gamma>0$, the two sets $\mathscr{C}_{B}^{-}(\gamma)$ and $\mathscr{C}_{B}\left(\frac{1}{\gamma}\right)$ coincide, i.e.,

$$
\mathscr{C}_{B}^{-}(\gamma)=\mathscr{C}_{B}\left(\frac{1}{\gamma}\right)
$$

and the Nyquist diagram of the frequency responses $\mathscr{C}_{B \gamma}^{-}(j \omega)$ and $\mathscr{C}_{B \frac{1}{\gamma}}(j \omega)$ corresponding to $\mathscr{C}_{B \gamma}^{-}(s)$ and $\mathscr{C}_{B \frac{1}{\gamma}}(s)$, respectively, have the same shape.

The intersections $p_{1}$ and $p_{2}$ of $\mathscr{C}_{B \gamma}^{-}(j \omega)$ with the straight line $r$ passing through points 0 and $B$ are $p_{1}=B$ and $p_{2}=\frac{B}{\gamma}$, respectively. The corresponding graphical representation is shown in Fig. 3.

Proof: each element $\mathscr{C}_{B \gamma}^{-}(s)$ of the set $\mathscr{C}_{B}^{-}(\gamma)$ also belongs to the set $\mathscr{C}_{B}\left(\frac{1}{\gamma}\right)$. In fact, from (8), (12) and (13), it follows that

$$
\begin{aligned}
\mathscr{C}_{B \gamma}^{-}(s) & =\left.\left.B \frac{s^{2}+2 \delta \omega_{n} s+\omega_{n}^{2}}{s^{2}+2 \gamma \delta \omega_{n} s+\omega_{n}^{2}}\right|_{\substack{\forall \delta>0 \\
\forall \omega_{n}>0}} \frac{s^{2}+2\left(\frac{1}{\gamma}\right) \bar{\delta} \omega_{n} s+\omega_{n}^{2}}{s^{2}+2 \bar{\delta} \omega_{n} s+\omega_{n}^{2}}\right|_{\substack{\forall \bar{\delta}>0 \\
\forall \omega_{n}>0}} \in \mathscr{C}_{B}\left(\frac{1}{\gamma}\right),
\end{aligned}
$$

where $\bar{\delta}=\gamma \delta$. In the same way it can be easily proved that each element $\mathscr{C}_{B \frac{1}{\gamma}}(s)$ of $\mathscr{C}_{B}\left(\frac{1}{\gamma}\right)$ also belongs to $\mathscr{C}_{B}^{-}(\gamma)$, and therefore $\mathscr{C}_{B}^{-}(\gamma)$ and $\mathscr{C}_{B}\left(\frac{1}{\gamma}\right)$ coincide. Moreover, the shape of the Nyquist diagrams of $\mathscr{C}_{B \gamma}^{-}(s)$ and $\mathscr{C}_{B \frac{1}{\gamma}}(s)$ depend only on $\gamma$ and therefore they coincide. From (9) and (14) it follows that the Nyquist diagram of $\mathscr{C}_{B \gamma}^{-}(s)$ is a circle whose center $C_{0}=B(\gamma+1) /(2 \gamma)$ lies on the real axis, whose radius is $R_{0}=|\gamma-1| /(2 \gamma)$ and its intersections with the real axis occur at points $a=B$ and $b=\frac{B}{\gamma}$, see Fig. 3 .

Definition 4: (Inversion Formulae) given two points $A=M_{A} e^{j \varphi_{A}}$ and $B=M_{B} e^{j \varphi_{B}}$ of the complex plane $\mathbb{C}$, the inversion formulae $X(A, B)$ and $Y(A, B)$ are defined as

$$
\begin{aligned}
X(A, B) & =\frac{M-\cos \varphi}{\sin \varphi}, \\
Y(A, B) & =\frac{\cos \varphi-\frac{1}{M}}{\sin \varphi},
\end{aligned}
$$

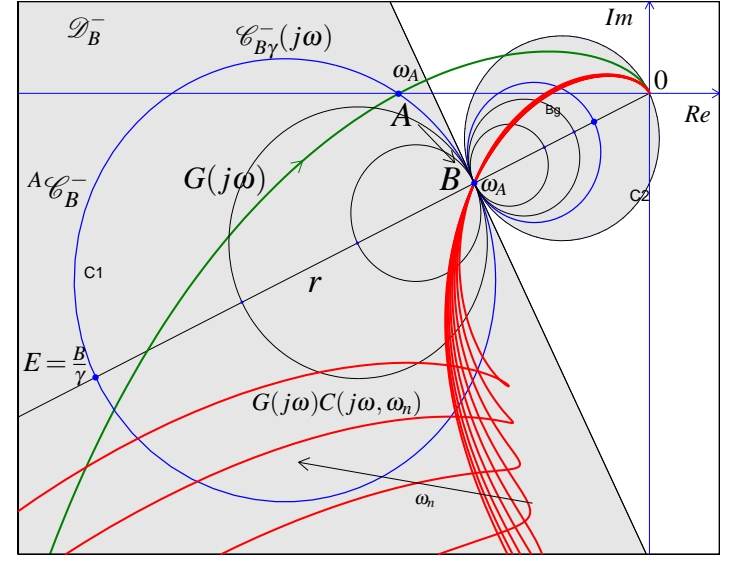

Fig. 4. Design of the lead-lag compensators $C\left(j \omega, \omega_{n}\right)$ moving point $A$ to point $B$.

where $M=\frac{M_{B}}{M_{A}}$ and $\varphi=\varphi_{B}-\varphi_{A}$.

These equations are the same inversion formulae for the continuous-time case introduced in [11] and used in [12] and [13].

Property 3: (From $A$ to $B$ ) given a point $B \in \mathbb{C}$ and chosen a point $A$ of the frequency response $G(j \omega)$ at frequency $\omega_{A}$ belonging to the controllable domain $\mathscr{D}_{B}^{-}$, i.e., $A=G\left(j \omega_{A}\right) \in \mathscr{D}_{B}^{-}$, the set $C\left(s, \omega_{n}\right)$ of all the lead-lag compensators $C(s)$ that move point A to point B is obtained from (1) using the parameters

$$
\gamma=\frac{X}{Y}>0, \quad \delta=Y \frac{\omega_{n}^{2}-\omega_{A}^{2}}{2 \omega_{n} \omega_{A}}>0
$$

for all $\omega_{n}>0$ such that $\delta>0$ and with parameters $X=X(A, B)$ and $Y=Y(A, B)$ obtained using the inversion formulae (15).

Proof: For $\omega=\omega_{A}$, (5) can be rewritten as

$$
\gamma=\frac{X\left(\omega_{A}\right)}{Y\left(\omega_{A}\right)}, \quad \delta=Y\left(\omega_{A}\right) \frac{\omega_{n}^{2}-\omega_{A}^{2}}{2 \omega_{n} \omega_{A}} .
$$

Substituting in (1) yields

$$
C\left(s, \omega_{n}\right)=\frac{s^{2}+X\left(\omega_{A}\right) \frac{\omega_{n}^{2}-\omega_{A}^{2}}{\omega_{A}} s+\omega_{n}^{2}}{s^{2}+Y\left(\omega_{A}\right) \frac{\omega_{n}^{2}-\omega_{A}^{2}}{\omega_{A}} s+\omega_{n}^{2}} .
$$

The frequency response of $C\left(s, \omega_{n}\right)$ at frequency $\omega_{A}$ is equal to the constant value

$$
C\left(j \omega_{A}, \omega_{n}\right)=C\left(j \omega_{A}\right)=\frac{1+j X}{1+j Y} .
$$

From (10) and (11) it is evident that point $A=$ $G\left(j \omega_{A}\right)=M_{A} e^{j \varphi_{A}}$ can be moved to point $B=$ $M_{B} e^{j \varphi_{B}}$ if and only if

$$
C\left(j \omega_{A}\right)=M e^{j \varphi}=\frac{M_{B}}{M_{A}} e^{j\left(\varphi_{B}-\varphi_{A}\right)} .
$$

From (17) and (18) we obtain the inversion formulae $X=X(A, B)$ and $Y=Y(A, B)$ introduced 
in Definition 4. The hypothesis that point $A$ belongs to the controllable domain $\mathscr{D}_{B}^{-}$ensures, see Definition 2, that there exist admissible lead-lag controllers $C\left(s, \omega_{n}\right)$ moving point $A$ to point $B$ which are characterized by positive parameters $\gamma$, $\delta$ and $\omega_{n}$. All the admissible values of $\omega_{n}$ are those which in (16) satisfy $\delta>0$.

\section{Design Problem $\left(\phi_{m}, G_{m}\right)$ And GRAPHICAL REPRESENTATIONS.}

Design Problem $\left(\phi_{m}, G_{m}\right)$ : given the control scheme of Fig. 2, the transfer function $G(s)$ and the design specifications on the phase margin $\phi_{m}$ and gain margin $G_{m}$, design a lead-lag compensator $C(s)$ such that the loop gain transfer function $C(j \omega) G(j \omega)$ passes through points $B_{p}=e^{j\left(\pi+\phi_{m}\right)}$ and $B_{g}=-1 / G_{m}$.

Solution: let $B_{p}=e^{j\left(\pi+\phi_{m}\right)}$ and $B_{g}=-1 / G_{m}=$ $M_{B_{g}} e^{j \varphi_{B g}}$ denote the points corresponding to the desired phase margin $\phi_{m}$ and gain margin $G_{m}$. The set $C_{\gamma}\left(s, \omega_{p}, \omega_{g}\right)$ of all the compensators $C(s)$ which solve this Design Problem is obtained as follows:

a) find all the pairs $\left(\omega_{p}, \omega_{g}\right) \in S_{\gamma \omega}$ of frequencies which solve the equation

$$
\gamma=\gamma_{p}\left(\omega_{p}\right)=\gamma_{g}\left(\omega_{g}\right),
$$

where the gain $\gamma>0$ is chosen arbitrarily, $S_{\gamma \omega}$ is the set of all the pairs $\left(\omega_{p}, \omega_{g}\right)$ satisfying (19), and functions $\gamma_{p}\left(\omega_{p}\right)$ and $\gamma_{g}\left(\omega_{g}\right)$ are defined as

$$
\gamma_{p}\left(\omega_{p}\right)=\frac{X_{p}}{Y_{p}}, \quad \gamma_{g}\left(\omega_{g}\right)=\frac{X_{g}}{Y_{g}},
$$

where the coefficients $X_{p}=X\left(A_{p}\left(\omega_{p}\right), B_{p}\right), Y_{p}=$ $Y\left(A_{p}\left(\omega_{p}\right), B_{p}\right), \quad X_{g}=X\left(A_{g}\left(\omega_{g}\right), B_{g}\right)$ and $Y_{g}=$ $Y\left(A_{g}\left(\omega_{g}\right), B_{g}\right)$ are obtained using the inversion formulae (15), $A_{p}=G\left(j \omega_{p}\right)=M_{A_{p}}\left(\omega_{p}\right) e^{j \varphi_{A_{p}}\left(\omega_{p}\right)}$ and $A_{g}=G\left(j \omega_{g}\right)=M_{A_{g}}\left(\omega_{g}\right) e^{j \varphi_{A g}\left(\omega_{g}\right)}$.

b) for each pair $\left(\omega_{p}, \omega_{g}\right) \in S_{\gamma \omega}$ compute

$$
\begin{gathered}
\omega_{n}=\sqrt{\frac{X_{g} \omega_{g}-X_{p} \omega_{p}}{\frac{X_{g}}{\omega_{g}}-\frac{X_{p}}{\omega_{p}}}}=\sqrt{\frac{Y_{g} \omega_{g}-Y_{p} \omega_{p}}{\frac{Y_{g}}{\omega_{g}}-\frac{Y_{p}}{\omega_{p}}}}>0, \\
\delta=Y_{p} \frac{\omega_{n}^{2}-\omega_{p}^{2}}{2 \omega_{n} \omega_{p}}=Y_{g} \frac{\omega_{n}^{2}-\omega_{g}^{2}}{2 \omega_{n} \omega_{g}}>0 .
\end{gathered}
$$

A solution $C_{\gamma}\left(s, \omega_{p}, \omega_{g}\right)$ of Design Problem $\left(\phi_{m}, G_{m}\right)$ exists only if: 1) $\gamma$ satisfies

$$
0<\gamma<\min \left[\max \left(\gamma_{p}\left(\omega_{p}\right)\right), \max \left(\gamma_{g}\left(\omega_{g}\right)\right)\right]
$$

2) $S_{\gamma \omega}$ is not empty; 3) points $A_{p}\left(\omega_{p}\right)$ and $A_{g}\left(\omega_{g}\right)$ belong, respectively, to the controllable domains $\mathscr{D}_{B_{p}}^{-}$and $\left.\mathscr{D}_{B_{g}}^{-} ; 4\right)$ parameters $\omega_{n}$ in $(21)$ and $\delta$ in (22) are real and positive.

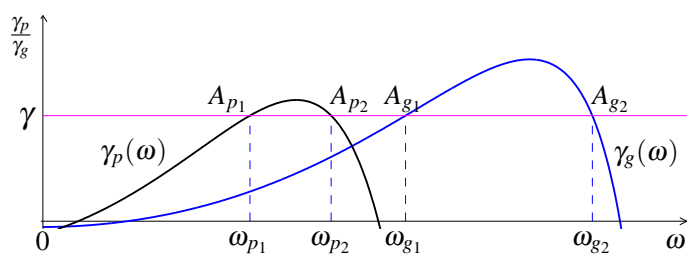

Fig. 5. Functions $\gamma_{g}(\omega)$ (blue line) and $\gamma_{p}(\omega)$ (black line) intersect the value $\gamma$ (magenta line) at frequencies $\left\{\omega_{g 1}, \omega_{g 2}\right\}$ and $\left\{\omega_{p 1}, \omega_{p 2}\right\}$, respectively.

Proof: the design specifications on the phase margin $\phi_{m}$ and gain margin $G_{m}$ completely define the position of points $B_{p}$ and $B_{g}$ on the complex plane. A solution $C_{\gamma}\left(s, \omega_{p}, \omega_{g}\right)$ exists only if the frequencies $\omega_{p}$ and $\omega_{g}$ satisfy

$$
G\left(j \omega_{p}\right) C_{\gamma}\left(j \omega_{p}\right)=B_{p}, \quad G\left(j \omega_{g}\right) C_{\gamma}\left(j \omega_{g}\right)=B_{g},
$$

that is only if $C(s)$ moves point $A_{p}=G\left(j \omega_{p}\right)$ to point $B_{p}$ and point $A_{g}=G\left(j \omega_{g}\right)$ to point $B_{g}$. The first condition is verified when $\gamma$ and $\delta$ are obtained from Property 3 when $\omega_{A}=\omega_{p}, A=A_{p}$ and $B=B_{p}$, the second when $\omega_{A}=\omega_{g}, A=A_{g}$ and $B=B_{g}$ :

$$
\begin{aligned}
& \gamma=\gamma_{p}\left(\omega_{p}\right)=\frac{X_{p}}{Y_{p}}, \quad \delta=Y_{p} \frac{\omega_{n}^{2}-\omega_{p}^{2}}{\omega_{p}}, \\
& \gamma=\gamma_{g}\left(\omega_{g}\right)=\frac{X_{g}}{Y_{g}}, \quad \delta=Y_{g} \frac{\omega_{n}^{2}-\omega_{g}^{2}}{\omega_{g}}
\end{aligned}
$$

where the coefficients $X_{p}=X\left(A_{p}\left(\omega_{p}\right), B_{p}\right), Y_{p}=$ $Y\left(A_{p}\left(\omega_{p}\right), B_{p}\right), \quad X_{g}=X\left(A_{g}\left(\omega_{g}\right), B_{g}\right)$ and $Y_{g}=$ $Y\left(A_{g}\left(\omega_{g}\right), B_{g}\right)$ are obtained using the inversion formulae (15). Relations (25) and (26) lead directly to (19) and (22). Solving (22) with respect to $\omega_{n}$ leads to the expression of $\omega_{n}$ given in (21). For each value of $\gamma$ satisfying (23), one can find the set $S_{\gamma \omega}$ of all the solutions $\left(\omega_{p}, \omega_{g}\right)$ of (19). This relation does not depend on $\delta$ and $\omega_{n}$, but only on the pairs $\left(\omega_{p}, \omega_{g}\right)$ and points $\left(B_{p}, B_{g}\right)$. Each solution $\left(\omega_{p}, \omega_{g}\right) \in S_{\gamma \omega}$ corresponds to an acceptable regulator $C_{\gamma}\left(s, \omega_{p}, \omega_{g}\right)$ only if points $A_{p}$ and $A_{g}$ belong respectively to the controllable domains $\mathscr{D}_{B_{p}}^{-}$and $\mathscr{D}_{B_{g}}^{-}$, according to Definition 2, and $\omega_{n}$ and $\delta$ given in (21) and (22) are real and positive, as assumed in (1).

The numerical solution of (19) can be obtained graphically by plotting $\gamma_{p}(\omega)$ and $\gamma_{g}(\omega)$ and by finding, for each admissible value of $\gamma$, all the pairs $\left(\omega_{p}, \omega_{g}\right) \in S_{\omega_{p}}$ where $\gamma_{p}\left(\omega_{p}\right)$ and $\gamma_{g}\left(\omega_{g}\right)$ intersect the horizontal line $\gamma$, see Fig. 5. In the example of Fig. 5 there are four different solutions: $S_{\gamma \omega}=\left\{\left(\omega_{p 1}, \omega_{g 1}\right),\left(\omega_{p 1}, \omega_{g 2}\right),\left(\omega_{p 2}, \omega_{g 1}\right),\left(\omega_{p 2}, \omega_{g 2}\right)\right\}$. The loop gain frequency responses $H_{11}(s), H_{12}(s)$, $H_{21}(s)$ and $H_{22}(s)$ of these four solutions on the Nyquist plane are shown in Fig. 6. These solutions are acceptable only if constraints $\delta>0$ and $\omega_{n}>0$ given in (21) and (22) are satisfied. 


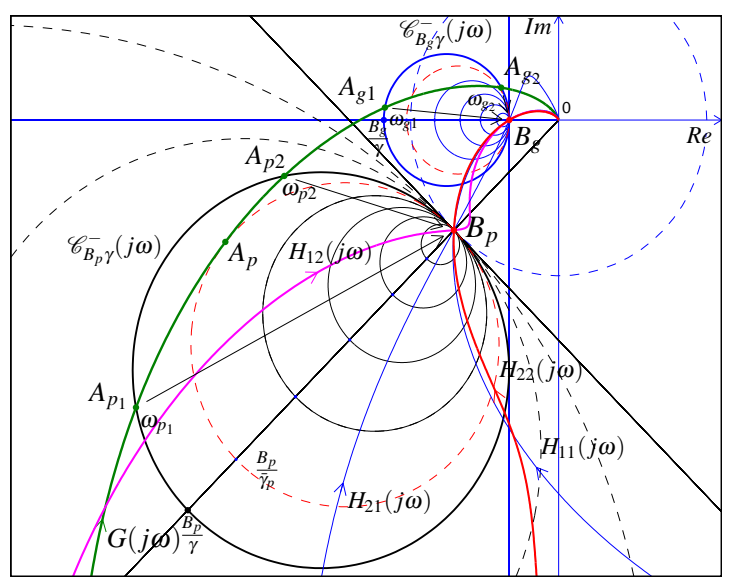

Fig. 6. Graphical determination of frequencies $\left(\omega_{p}, \omega_{g}\right)$ on the Nyquist plane. Loop gain frequency response $H_{11}(s), H_{12}(s)$, $H_{21}(s)$ and $H_{22}(s)$ satisfy desired phase and gain margin.

\section{A. Graphical Solution on the Nyquist plane.}

The graphical solution of (19) can also be obtained using the graphical construction shown in Fig. 6:

1) given points $B_{p}$ and $B_{g}$ and a desired value $\gamma>0$, draw the circles having their diameters on the segments $\left(B_{p}, B_{p} / \gamma\right)$ and $\left(B_{g}, B_{g} / \gamma\right)$. From Property 2 they coincide with the frequency responses $\mathscr{C}_{B_{p} \gamma}^{-}(j \omega)$ and $\mathscr{C}_{B_{g} \gamma}^{-}(j \omega)$.

2) if the frequency response $G(j \omega)$ does not intersect both circles $\mathscr{C}_{B_{p} \gamma}^{-}(j \omega)$ and $\mathscr{C}_{B_{g} \gamma}^{-}(j \omega)$, the chosen value of $\gamma$ is not acceptable.

3) otherwise, each pair $\left(\omega_{p}, \omega_{g}\right)$ corresponding to the intersections of $G(j \omega)$ with circles $\mathscr{C}_{B_{p}}^{-}(j \omega)$ and $\mathscr{C}_{B_{g} \gamma}^{-}(j \omega)$ is a possibile solution for the Design Problem $\left(\phi_{m}, G_{m}\right)$.

This graphical construction hinges on the fact that frequencies $\omega_{p}$ and $\omega_{g}$ satisfy (24), which can be rewritten as

$$
\left\{\begin{array}{l}
\left.G(j \omega)\right|_{\omega=\omega_{p}}=\frac{B_{p}}{C_{\gamma}\left(j \omega_{p}\right)}=\left.\mathscr{C}_{B_{p} \gamma}^{-}(j \omega)\right|_{\omega=\omega_{p}} \\
\left.G(j \omega)\right|_{\omega=\omega_{g}}=\frac{B_{g}}{C_{\gamma}\left(j \omega_{g}\right)}=\left.\mathscr{C}_{B_{g} \gamma}^{-}(j \omega)\right|_{\omega=\omega_{g}}
\end{array}\right.
$$

The Solution of the Design Problem $\left(\phi_{m}, G_{m}\right)$ is also useful for solving, for example, the following Design Problems:

A) Design a lead-lag compensator $C(s)$ which satisfies constraints on the phase margin $\phi_{m}$, the gain margin $G_{m}$ and the gain crossover frequency $\omega_{p}$. Solution: $\gamma=\frac{X_{p}}{Y_{p}}$ in (20) is determined by points $B_{p}=e^{j\left(\pi+\phi_{m}\right)}$ and $A_{p}=G\left(j \omega_{p}\right)$, the values of $\omega_{g} \in S_{\gamma \omega}$ are obtained from (19) solving the equation $\gamma=\gamma_{g}\left(\omega_{g}\right)$, parameters $\omega_{n}$ and $\delta$ are given by relations (21) and (22).

B) Design a lead-lag compensator $C(s)$ which satisfies constraints on the phase margin $\phi_{m}$, the gain margin $G_{m}$ and the phase crossover frequency $\omega_{g}$. Solution: $\gamma=\frac{X_{g}}{Y_{g}}$ in (20) is determined by points $B_{g}=-1 / G_{m}$ and $A_{g}=G\left(j \omega_{g}\right)$, the values of $\omega_{p} \in S_{\gamma \omega}$ are obtained from (19) solving equation $\gamma=\gamma_{p}\left(\omega_{p}\right)$, parameters $\omega_{n}$ and $\delta$ are given by relations (21) and (22).

C) Design a lead-lag compensator $C(s)$ which satisfies constraints on the phase margin $\phi_{m}$, the gain margin $G_{m}$ and the maximum allowable gain $\gamma$. Solution: $\gamma$ is the minimum of the two values $\bar{\gamma}_{p}$ and $\bar{\gamma}_{g}$ that can be graphically determined on the Nyquist plane by drawing the circles $\mathscr{C}_{B_{p}}^{-} \gamma_{p}(j \omega)$ and $\mathscr{C}_{B_{g} \gamma_{g}}^{-}(j \omega)$ tangent to function $G(j \omega)$, see Fig. 6. The same values $\bar{\gamma}_{p}$ and $\bar{\gamma}_{g}$ can also be determined by finding the maximum points of the functions $\gamma_{p}\left(\omega_{p}\right)$ and $\gamma_{g}\left(\omega_{g}\right)$ defined in (20), see Fig. 5. The frequencies $\omega_{p}$ and $\omega_{g}$ corresponding to value $\gamma=\min \left(\bar{\gamma}_{p}, \bar{\gamma}_{g}\right)$ can be substituted in (21) and (22) providing the parameters $\omega_{n}$ and $\delta$ of the lead-lag compensator $C(s)$.

D) Design a lead-lag compensator $C(s)$ which satisfies the gain margin $G_{m}$, the phase crossover frequency $\omega_{g}$ and the maximum allowable phase margin $\phi_{m}$. Solution: $\gamma=\frac{X_{g}}{Y_{g}}$ in (20) is determined by points $B_{g}=-1 / G_{m}$ and $A_{g}=G\left(j \omega_{g}\right)$. The value of $\gamma$ completely defines the shape of circle $\mathscr{C}_{B \frac{1}{\gamma}}(j \omega)$. The maximum value $\bar{\phi}_{m}$ of the phase margin $\phi_{m}$ can be graphically determined moving point $B_{p}=e^{j\left(\pi+\phi_{m}\right)}$ on the unit circle until $\mathscr{C}_{B \frac{1}{\gamma}}(j \omega)$ is tangent to function $G(j \omega)$. The frequency $\omega_{p}$ of the tangent point substituted in (21) and (22) provides the parameters $\omega_{n}$ and $\delta$ of the compensator $C(s)$.

\section{NUMERICAL EXAMPLES}

The graphical representations shown in Fig. 5 and Fig. 6 have been obtained referring to the following transfer function:

$$
G(s)=\frac{1200(s+2)}{(s+1.5)^{2}(s+7)^{2}}
$$

and solving the Design Problem $\left(\phi_{m}, G_{m}\right)$ subject to the following specifications: $\phi_{m}=45^{\circ}, G_{m}=3$ and $\gamma=0.282$.

The solutions of the Design Problem have been obtained as follows. The design specifications define points $B_{p}=e^{j 225^{\circ}}$ and $B_{g}=0.333 e^{j 180^{\circ}}$. The four solutions $\left(\omega_{p i}, \omega_{g j}\right)$ of (19) can be graphically determined as shown in Fig. 5: $\omega_{p i}=\{4.82,6.72\}, \omega_{g j}=\{8.45,12.8\}$. The corresponding points on function $G(j \omega)$ are $A_{p 1}=$ $3.4 e^{-j 147.1^{\circ}}, A_{p 2}=1.89 e^{-j 169^{\circ}}, A_{g 1}=1.17 e^{j 176.1^{\circ}}$ and $A_{g 2}=0.44 e^{j 151.8^{\circ}}$. By substitution in (21) and (22) we obtain only two acceptable and stable 


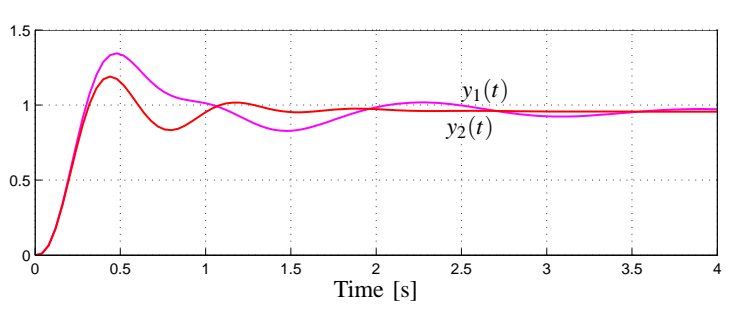

Fig. 7. Step responses $y_{1}(t)$ (magenta line) and $y_{2}(t)$ (red line) of the closed loop system when, respectively, regulators $C_{\gamma}\left(s, \omega_{p 1}, \omega_{g 2}\right)$ in (29) and $C_{\gamma}\left(s, \omega_{p 2}, \omega_{g 2}\right)$ in are used.

regulators $C_{\gamma}\left(s, \omega_{p}, \omega_{g}\right)$. The first one is obtained for $\left(\omega_{p 1}, \omega_{g 2}\right), \delta=1.21$ and $\omega_{n}=4.345$ :

$$
C_{\gamma}\left(s, \omega_{p 1}, \omega_{g 2}\right)=\frac{s^{2}+2.97 s+18.88}{s^{2}+10.5 s+18.88} .
$$

The second one is obtained for $\left(\omega_{p 2}, \omega_{g 2}\right), \delta=2.90$ and $\omega_{n}=1.99$ :

$$
C_{\gamma}\left(s, \omega_{p 1}, \omega_{g 2}\right)=\frac{s^{2}+3.27 s+3.98}{s^{2}+11.58 s+3.98} .
$$

The other solutions are not acceptable because for them the constraints $\omega_{n}>0$ and $\delta>0$ are not satisfied. The Nyquist plots of the loop gain transfer functions $H_{i j}(j \omega)=C_{\gamma}\left(j \omega, \omega_{p i}, \omega_{g j}\right) G(j \omega)$ are shown in Fig. 6. The step responses of the acceptable solutions are shown in Fig. 7.

Solution of the Design Problem $C$ : the maximum $\gamma=\bar{\gamma}_{p}$ which satisfies the Design Problem $C$ when $\phi_{m}=45^{\circ}$ and $G_{m}=3$ can be graphically determined drawing the circle $\mathscr{C}_{B_{p} \gamma_{p}}^{-}(j \omega)$ tangent in $A_{p}$ to function $G(j \omega)$ as shown in Fig. 6. The obtained value is $\bar{\gamma}_{p}=0.324$.

Solution of the Design Problem D: given the phase crossover frequency $\omega_{g 2}=12.8$ and the gain margin $G_{m}=3$, the maximum phase margin $\bar{\phi}_{m}$ which satisfies the Design Problem $D$ can be graphically determined as shown in Fig. 8. The obtained value is $\bar{\phi}_{m}=51.75^{\circ}$. When phase margin $\phi_{m}$ increases from $40^{\circ}$ to $\bar{\phi}_{m}$, function $\gamma_{p}(\omega)$ modifies its shape as shown in Fig. 9.

\section{Vi. Conclusion}

A general form of lead-lag compensators which includes real or complex zeros and poles has been considered. Necessary and sufficient conditions to exactly satisfy gain and phase margins specifications have been proposed together with a simple graphical interpretation on the Nyquist plane. The presented results seem to be useful both for educational and industrial purposes.

\section{REFERENCES}

[1] Weng Khuen Ho, Chang Chieh Hang and Lisheng S. Cao, Tuning of PID controllers based on gain and phase margin specifications. Automatica: 31(3):497-502, 1995.

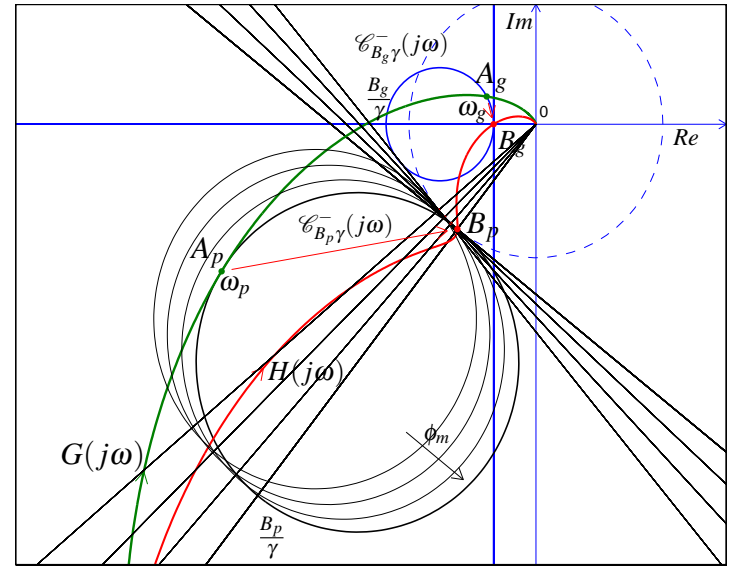

Fig. 8. Graphical solution of Design Problem D.

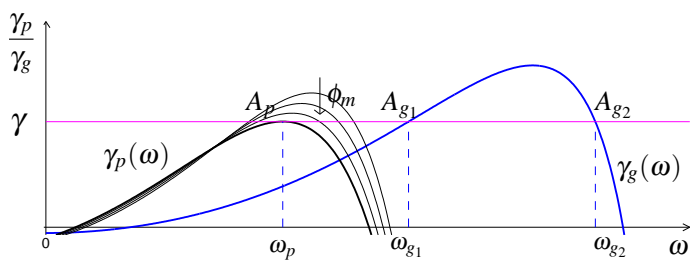

Fig. 9. Design problem $D$ : function $\gamma_{g}(\omega)$ (blue line) and functions $\gamma_{p}(\omega)$ (black lines) for $\phi_{m}=\{40,44,48,51.75\}$.

[2] K.S. Yeung, K.W. Wong and K.L. Chen, A Non Traialand-Error Method for Lag-Lead Compensator Design. IEEE Transactions on Education, E-41, no. 1, Feb. 1998.

[3] K.J. Aström and T. Hagglund, Automatic Tuning of Simple Regulators with Specifications on Phase and Amplitude Margins. Automatica: Vol. 20,No.5, 645-651, Sep. 1984.

[4] Wang Qing-Guo, Fung Ho-Wang, Zhang Yu, PID tuning with exact gain and phase margins. ISA Transactions, 38,243-249, 1999.

[5] Fung Ho-Wang, Wang Qing-Guo and Lee Tong-Heng, PI tuning in terms of gain and phase margins. Automatica: 34(9):1145-9, 1998

[6] Ho WK, Gan OP, Tay EB, Ang EL, Performance and gain and phase margins of well-known PID tuning formulas. IEEE Transactions on Control Systems Technology: 4:473-7, 1996.

[7] Lee Ching-Hung, A survey of PID controller design based on gain and phase margins. International Journal of Computational Cognition: 2(3):63-100, 2004.

[8] W.C. Messner, M.D. Bedillion, L. Xia and D.C. Karns. Lead and Lag Compensators with Complex Poles and Zeros: design formulas for modeling and loop shaping. IEEE Control Systems Magazine, vol. 27, no. 1, pp.4454, Feb 2007.

[9] S.S. Flores, A.M. Valle and B.A. Castillejos, Geometric Design of Lead/Lag Compensators Meeting a Hinf Specification. 4rd ICEEE, Mexico City, Mexico, September 5-7, 2007.

[10] W. Messner, Formulas for Asymmetric Lead and Lag Compensators. American Control Conference, St. Louis, MO, USA, June 10-12, 2009.

[11] Charles L. Phillips. Analytical Bode Design of Controllers. IEEE Transactions on Education, E-28, no. 1, pp. 43-44, 1985.

[12] G. Marro and R. Zanasi, "New Formulae and Graphics for Compensator Design", IEEE International Conference On Control Applications, Trieste, Italy, September 1-4, 1998.

[13] R. Zanasi, R.Morselli, "Discrete Inversion Formulas for the Design of Lead and Lag Discrete Compensators", ECC, 23-26 August 2009, Budapest, Hungary. 